\title{
Metastatic mesonephric adenocarcinoma of unknown origin after hysterectomy: A case report
}

\author{
Youngsun Kim ${ }^{1}$, Sungkyoung $\mathrm{Moon}^{2}$, Kiyong $\mathrm{Na}^{3}$ \\ ${ }^{1}$ Department of Obstetrics and Gynecology, Kyung Hee Medical Center, College of Medicine, Kyung Hee University, Seoul, Republic of Korea \\ ${ }^{2}$ Department of Radiology, Kyung Hee Medical Center, College of Medicine, Kyung Hee University, Seoul, Republic of Korea \\ ${ }^{3}$ Department of Pathology, Kyung Hee Medical Center, College of Medicine, Kyung Hee University, Seoul, Republic of Korea
}

\begin{abstract}
Summary
Müllerian and Wolffian ducts originate from the mesodermal tissue. In females, the Müllerian ducts develop to form the fallopian tubes, uterus, cervix, and upper two-thirds of the vagina; whereas in males, they regress. The Wolffian duct, also called the mesonephric duct, regresses in females during development. Mesonephric carcinomas occur through the female genital tract, and the majority arise in the cervix of the uterus. We report the case of a patient who had abdominal pain 4 weeks prior to admission, and who underwent a hysterectomy 20 years prior. The radiologic results revealed a $6.7 \times 5.4 \times 4.3 \mathrm{~cm}$-sized mass in the retroperitoneum but were not indicative of mesonephric adenocarcinoma. The patient underwent complete mass surgical resection, followed by posterior sectionectomy of the liver and splenectomy with cholecystectomy. The histopathologic results showed that the mass was a mesonephric adenocarcinoma
\end{abstract}

Key words: Hysterectomy; Mesonephric adenocarcinoma; Metastatic; Pelvic mass; Unknown origin.

\section{Introduction}

The mesonephric duct regresses in females during development [1], and remnants of the duct may persist in the cervix, vagina, adnexa, and uterine corpus [2]. Mesonephric carcinomas occur through the female genital tract and most of them arise in the cervix of uterus [1]. Mesonephric adenocarcinoma of the cervix is a rare tumor derived from the remnants of the mesonephric duct [3]. Differential diagnosis from other cervical carcinomas is difficult because little is known about its biological behavior, prognosis, and management [3]. The majority of mesonephric adenocarcinomas occur in the cervix, and mesonephric adenocarcinoma of the vagina is extremely rare, with only a few cases reported in the literature. Consequently, there is no established standard treatment, and the prognosis and biological behavior of vaginal mesonephric adenocarcinoma remains largely unknown [4].

In our case, the patient underwent total hysterectomy 20 years prior, but the histopathologic results of the pelvic mass were mesonephric adenocarcinoma of unknown origin.

Herein, we present a rare case of mesonephric adenocarcinoma of unknown origin and treatment. This study was approved by the Ethics Committee and Institutional Review Board of Kyung Hee University Hospital (KHUH 2019-11029-007). The patient provided informed consent for publication of this case report.

\section{Case Report}

A 64-year-old woman presented to the Obstetrics and Gynecology department of our hospital with complaints of a pelvic mass and right hydronephrosis. The patient had no indications until 4 weeks before this presentation, when she began to notice an increased frequency of abdominal pain. She underwent a total abdominal hysterectomy and salpingo-oophorectomy 20 years prior because of multiple myoma of the uterus and a postmenopausal state.

Upon examination, the patient appeared healthy, and her vital signs were as follows: Temperature, $35.6{ }^{\circ} \mathrm{C}$; pulse, 80 beats per minute; blood pressure, $110 / 80 \mathrm{mmHg}$; respiratory rate, 20 breaths per minute; and oxygen saturation, 98\% (while breathing ambient air). The patient's abdomen was soft, without distention or tenderness, and her vagina appeared normal, with scant white discharge observed during pelvic examination. At that time, she underwent blood and radiological testing. The blood test results are presented in Table 1.

A cystic and solid mass in the right pelvic cavity was visualized via magnetic resonance imaging (MRI) of the pelvis. The mass abutted on the right corner of the vaginal stump and the right distal ureter (Figure 1A). The size of this retroperitoneal multi-loculated cystic mass was $6.7 \times$ $5.4 \times 4.3 \mathrm{~cm}$. As the right distal ureter was obstructed by extrinsic compression from the mass, the upstream ureter was diffusely dilated. The mass contained multiple cystic locules with visible fluid-fluid levels and an irregular solid portion, as observed on T2-weighted imaging (T2WI; Figure 1B). The cystic locules showed a high signal in-
Eur. J. Gynaecol. Oncol. - ISSN: 0392-2936 XLI, n. 5, 2020

doi: $10.31083 /$ j.ejgo.2020.05.5474
This is an open access article under the CC BY-NC 4.0 license (https://creativecommons.org/licenses/by-nc/4.0/). 

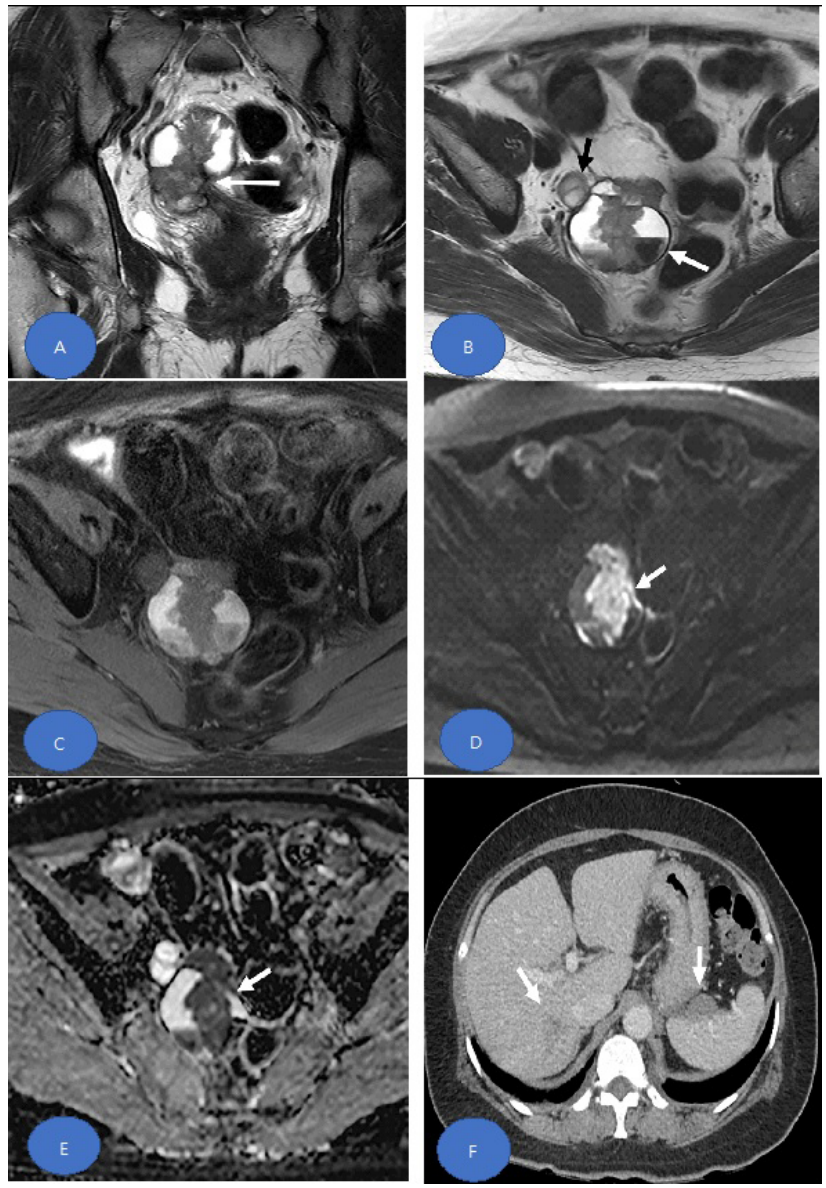

Figure 1. - Imaging studies of the abdomen and pelvis. Pelvic magnetic resonance imaging revealed a retroperitoneal multiloculated cystic and solid lesion measuring $6.7 \times 5.4 \times 4.3 \mathrm{~cm}$. The mass bordered the right corner of the vaginal stump and the right distal ureter (Panel A, arrow). The mass contained multiple cystic locules with visible fluid-fluid levels and an irregular solid portion (Panel B, arrows). The cystic locules produced a high signal intensity with fat suppression (Panel C). The solid portion showed marked diffusion restriction via diffusion-weighted imaging (Panel D and E, arrow). Additional soft tissue masses were observed in the liver and spleen via CT (Panel F, arrows).

tensity on fat suppression T1-weighted imaging (T1WI), which was indicative of internal hemorrhage along with the the fluid-fluid levels observed on T2WI (Figure 1C). The solid portion showed marked diffusion restriction via diffusion-weighted imaging and an apparent diffusion coefficient map with a b-value of 1000 (Figure 1D and 1E). The solid portion also showed considerable enhancement of T1WI after intravenous contrast enhancement. Because the solid portion was found to have high cellularity, it was considered to be either a malignant tumor arising from the vaginal stump or a metastatic tumor from an unknown primary cancer source.

Additional soft tissue masses were observed in the liver and spleen via computed tomography (CT; Figure 1F). A hypoattenuating solid mass with an irregular shape was lo- cated in liver segment 7 that extended along the right portal vein branches. Another soft tissue mass was located in the splenic hilum. These were considered to be the metastatic masses. Two days after admission, the patient underwent kidney scintigraphy using Tc-99 m DMSA $5 \mathrm{mCi}$. The results revealed no uptake in the right kidney, and the left kidney had a normal volume with no cortical defects. A double-J catheter was inserted into the right ureter owing to right hydronephrosis. Only mass excision surgery was performed because the radiologic results indicated a malignant tumor and metastasis. However, since all the tumor markers were within normal range, we believed that the mass was benign.

Upon histological examination, the tumor was found to have features of mesonephric adenocarcinoma arising in the retroperitoneum. The tumors showed diverse architectural patterns, including tubule-glandular (Figure 2A), sex cord-like (Figure 2B), solid (Figure 2C), and papillary cystic (Figure 2D) foci. Some of the tumor glands contained luminal eosinophilic material (Figure 2E). There were foci of bland-looking dilated glands lined by flat cuboidal epithelium, suggestive of mesonephric remnants, in contrast to the surrounding tumor glands (Figure 2F). Immunohistochemistry of the tumor cells showed focal GATA-3 staining (Figure 2G), diffuse PAX-2 staining (Figure 2H), and negative ER staining (Figure 2I).

The patient underwent additional surgery, involving posterior sectionectomy of the liver and splenectomy with cholecystectomy, 2 weeks later in the Department of General Surgery of our hospital. The spleen revealed metastatic mesonephric adenocarcinoma in the hilum (Figure 2J and $2 \mathrm{~K})$. The liver revealed multiple dilated intrahepatic bile ducts containing brown pigmented stones and necrotic material and papillary proliferation of the dysplastic biliary epithelium, suggesting intraductal papillary neoplasm of the bile duct (Figure 2L). After surgery, taxol-carboplatin chemotherapy was performed at the 6-month follow-up.

\section{Discussion}

Mesonephric adenocarcinoma is a rare malignant tumor that originates from mesonephric remnants of the female genital tract [5]. To the best of our knowledge, fewer than 100 cases have been reported in the current literature. Its diagnosis is pathologically difficult because mesonephric adenocarcinoma may exhibit varying morphological patterns [6], with cases reported in the vagina, uterine corpus, cervix, urinary bladder, urethra, and urethral diverticulum [7]. In addition, a number of cases have been reported around mesonephric remnant sites, such as the broad ligaments, fallopian tubes, and ovarian hila [7].

Histopathologic results may display numerous architectural growth patterns and foci of bland-looking and dilated glands lined by flat cuboidal epithelium, suggestive of mesonephric remnants. Indeed, the essential features in our case have similarities to those of previous studies. However, compared with other reports, our case was unique in 


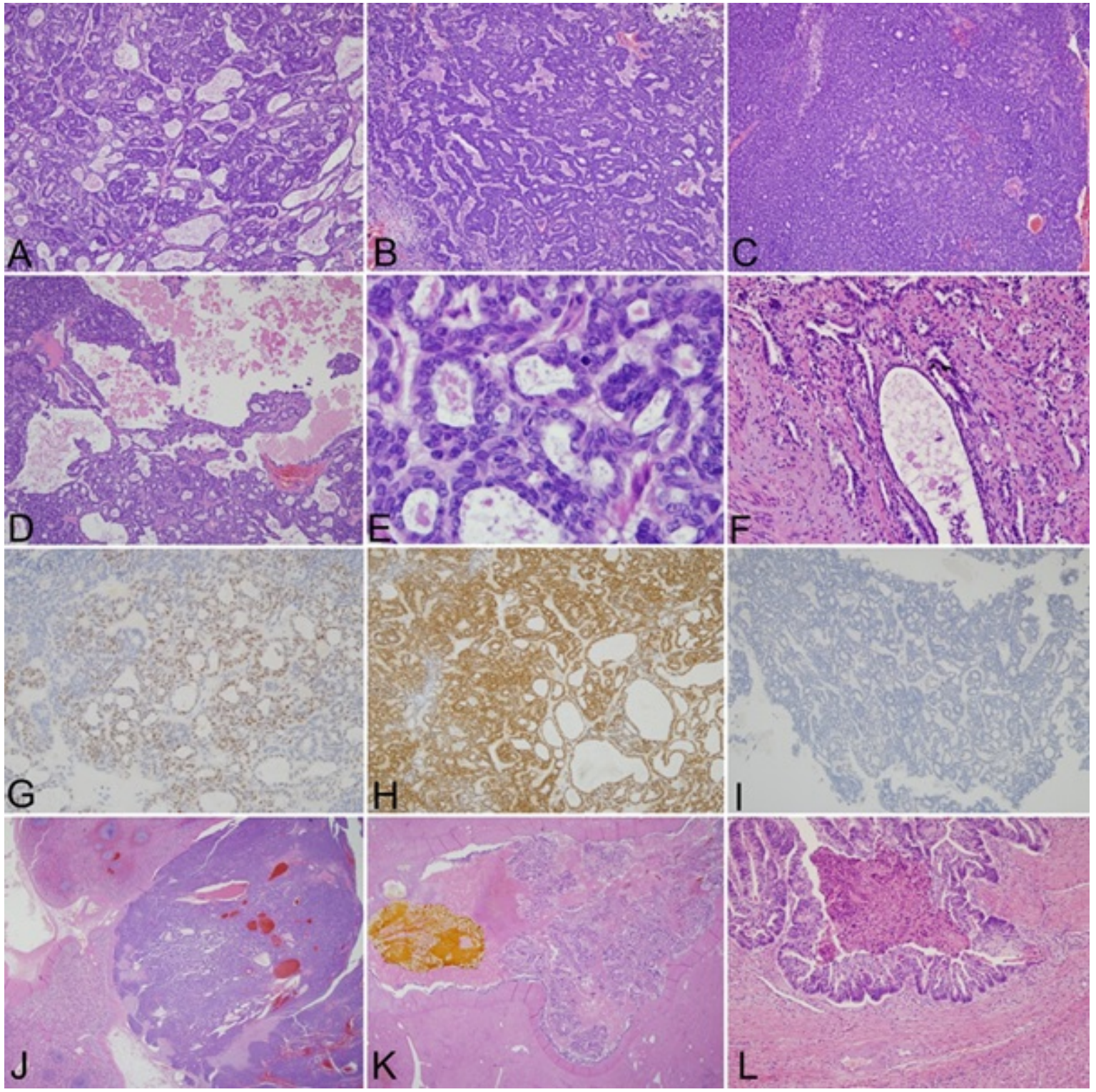

Figure 2. - Histopathologic findings of the pelvic mass. Representative histological features of the mesonephric adenocarcinoma arising in the retroperitoneum. The tumors show diverse architectural patterns, including tubule-glandular (Panel A), sex cord-like (Panel B), solid (Panel C), and papillary cystic (Panel D) foci. Some of the tumor glands contain luminal eosinophilic material (Panel E). The presence of foci is suggestive of mesonephric remnants, contrasting to the surrounding tumor glands (Panel F). Immunohistochemistry analysis of the tumor cells showed focal GATA-3 staining (Panel G), diffuse PAX-2 staining (Panel H), and negative ER staining (Panel I). Histopathologic evaluation of small masses in the spleen and liver (Panel J, K, and L).

that we did not know the origin of the mesonephric adenocarcinoma.

The mass contained multiple cystic locules with fluidfluid levels, and there were additional soft tissue masses observed in the liver and spleen via CT. The features of the masses were all highly suggestive of cancer. In the following sections, the general diagnostic approach to pelvic masses and the differential diagnosis of a suspected cancerous mass will be discussed.

Infectious causes of a pelvic mass are rare in older, postmenopausal women, and our patient did not present any risk factors for pelvic inflammatory disease. Moreover, she did not have a fever or systemic signs of infection but did present with abdominal pain. Her surgical history included a total hysterectomy and bilateral salpingo-oophorectomy. The absence of clinically significant abdominal tenderness, leukocytosis, or systemic signs of infection is not consistent with the signs of infection or an abscess. Although chronic infections (e.g., tuberculosis) can involve the pelvis, the patient had no travel history or chronic symptoms to support such a diagnosis [8].

Neoplastic diagnoses encompass common benign tu- 
Table 1. - Laboratory Data.

\begin{tabular}{lcc}
\hline Variable & Reference Range & On presentation \\
\hline White-cell count $\left(10^{3} / \mu \mathrm{L}\right)$ & $4.0-10.0$ & 7.88 \\
Differential count $(\%)$ & $0-4.5$ & 1.5 \\
Neutrophils & $40-74$ & 63.4 \\
Lymphocytes & $19-48$ & 25.3 \\
Monocytes & $4-9$ & 5.0 \\
Eosinophils & $0-7$ & 4.3 \\
Basophils & $0-1.5$ & 0.4 \\
Prothrombin time (sec) & $12.5-14.7$ & 12.7 \\
Prothrombin-time international normalized ratio (\%) & $0.9-1.2$ & 0.95 \\
Lactate dehydrogenase (U/L) & $218-472$ & 381 \\
CA-125 (U/mL) & $<35$ & 9.6 \\
SCC (ng/mL) & $<1.5$ & 0.6 \\
CEA (ng/mL) & $<4.1$ & 2.49 \\
Urine micro RBC (HPF) & $0-4$ & many \\
Urine micro WBC (HPF) & $0-4$ & $2-4$ \\
\hline
\end{tabular}

mors and a spectrum of malignant tumors, as well as some diagnoses that have features of both. Consequently, it is useful to consider blood tumor marker levels to distinguish between benign tumors and malignancy. The current patient previously underwent surgery of the uterus, both ovaries, and the salpinx; hence, we assumed that other benign tumors were present in the patient's pelvis. In this regard, we first considered bladder diverticulum, the cause of which is obstruction of the bladder outlet due to a mass or stricture [9]. Urethral diverticulum may occur in $0.6-6.0 \%$ of women [10], and the risk factors for urethral diverticulum are repeated infection of the peri-urethral glands and trauma from vaginal and urethral procedures [11]. Tail gut cysts are common benign tumors in the presacral region, although malignant transformation has been described [10]. Complete excision of the mass is necessary to prevent recurrence. Nerve sheath tumor (Schwannoma) is another benign tumor that arises from the sacral nerve root sheaths [10].

We also considered whether the patient had cancer. Urinary bladder cancer in women is increasing, although its incidence is 3-4 times lower than that in men [12].Unfortunately, bladder cancer is more often discovered at advanced stages in women [12]. Endocervical adenocarcinoma would be a reasonable consideration, had the patient not previously undergone total hysterectomy [2]. Moreover, carcinoembryonic antigen (CEA) is negative in mesonephric carcinoma, but is positive in endocervical adenocarcinoma [13]. Clear cell carcinoma, like mesonephric carcinoma, is HPV-negative; however, unlike mesonephric carcinoma, clear cell carcinoma has a prominent clear cytoplasm and may be related to diethylstilbestrol (DES) exposure in the uterus [2]. Rectal carcinoma and rectal gastrointestinal stromal tumor (GIST) were also considered; however, pelvic MRI revealed that these diagnoses were inappropriate.
For the final diagnosis, we performed mass excision with intraoperative frozen section examination. Two weeks after excision, the patient underwent posterior sectionectomy and splenectomy with cholecystectomy. It was initially believed that the patient had a benign tumor in her pelvis. However, the histopathological results revealed that it was actually a mesonephric adenocarcinoma; specifically, a metastatic mesonephric adenocarcinoma of the liver and spleen. In this case, the patient had had a hysterectomy 20 years prior, and the radiologic results were not indicative of mesonephric adenocarcinoma. The reason for this may be that the patient had metastatic lesions in the liver and spleen, although the origin of this cancer is unknown. Furthermore, it is possible that the cancer arose from a mesonephric duct remnant of the paracervix during the hysterectomy, as minimum resection of the paracervix was performed [14].

In conclusion, based on the findings of this case report, we can infer that maximum resection of the paracervix is preferred during total hysterectomy as far as possible.

\section{Ethics approval and consent to participate}

All subjects gave their informed consent for inclusion before they participated in the study. The study was conducted in accordance with the Declaration of Hesinki, and the protocol was approved by the Ethics Committee of Kyung Hee University Hospital (KHUH 2019-11-029. 007).

\section{Acknowledgments}

Thanks to all the peer reviewers and editors for their opinions and suggestions.

\section{Conflict of Interest}

The authors have no conflicts of interest to declare. 
Submitted: February 12, 2020

Accepted: April 01, 2020

Published: October 15, 2020

\section{References}

[1] Asano T, Yamamoto M.: "Mesonephric tumor, mesonephric carcinoma". Ryoikibetsu shokogun shirizu, 1997, 16, 512-515.

[2] Howitt B.E., Nucci M.R.: "Mesonephric proliferations of the female genital tract". Pathology (Phila.), 2018, 50, 141-150.

[3] Dierickx A., Göker M., Braems G., Tummers P., Van den Broecke R.: "Mesonephric adenocarcinoma of the cervix: Case report and literature review". Gynecologic Oncology Reports, 2016, 17, 7-11.

[4] Mueller I., Kametriser G., Jacobs V.R., Bogner G., Staudach A., Koch H., et al.: "Mesonephric adenocarcinoma of the vagina". Strahlenther. Onkol., 2016, 192, 668-671.

[5] Ferry J.A., Scully R.E.: "Mesonephric Remnants, Hyperplasia, and Neoplasia in the Uterine Cervix". the American Journal of Surgical Pathology, 1990, 14, 1100-1111.

[6] Montalvo N., Redrobán L., Galarza D.: "Mesonephric adenocarcinoma of the cervix: a case report with a three-year follow-up, lung metastases, and next-generation sequencing analysis". Diagn. Pathol., 2019, 14,71.

[7] Yao Y., Han J., Liu C.: "Mesonephric adenocarcinoma of the vaginal-urethral interspace". Chin Med J (Engl). 2014, 127, 984985.

[8] Curry A., Williams T., Penny M.L.: "Pelvic Inflammatory Dis- ease: Diagnosis, Management, and Prevention”. Am. Fam. Physician, 2019, 100, 357-364

[9] Li X., Liu T. and Wang L.: "Diagnosis of bladder diverticulum: Retrograde cystography CT more valuable". Journal of X-Ray Science and Technology, 2015, 23, 481-487.

[10] Gangadhar K., Mahajan A., Sable N., Bhargava P.: "Magnetic Resonance Imaging of Pelvic Masses: A Compartmental Approach". Seminars in Ultrasound, CT and MRI, 2017, 38, 213-230.

[11] Greenwell T.J., Spilotros M.: "Urethral diverticula in women". Nature Reviews Urology, 2015, 12, 671-680.

[12] Fankhauser C.D., Mostafid H.: "Prevention of bladder cancer incidence and recurrence". Curr. Opin. Urol., 2018, 28, 88-92.

[13] Lang G., Dallenbach-Hellweg G.: "The Histogenetic Origin of Cervical Mesonephric Hyperplasia and Mesonephric Adenocarcinoma of the Uterine Cervix Studied with Immunohistochemical Methods". Int. J. Gynecol. Pathol., 1990, 9, 145-157.

[14] Querleu D., Morrow C.P.: "Classification of radical hysterectomy". the Lancet Oncology, 2008, 9, 297-303.

Corresponding Author:

YOUNGSUN KIM

Department of Obstetrics and Gynecology

Kyung Hee Medical Center, College of Medicine,

Kyung Hee University, Kyungheedaero 23,

Dongdaemungu, Seoul (Korea).

e-mail: chacha0725@naver.com 